\title{
Effects of initial conditions on the Hoffman reflex
}

\author{
GERALD L. GOTTLIEB AND GYAN C. AGARWAL
}

From the Biomedical Engineering Department, Presbyterian St. Luke's Hospital, Graduate College at the Medical Center, University of Illinois, and the Department of Systems Engineering, University of Illinois a $\vec{R}$ Chicago Circle, Chicago, Illinois, U.S.A.

SUMMARY The Hoffmann reflex is a monosynaptic reflex elicited by electrical stimulation os afferent nerve fibres. The amplitude of the reflex response may be measured both by EMG recording from the muscle and by sensing the muscle twitch. These two effects are dependent, not only on the amplitude of the stimulus, but on the state of excitability of the afferent-efferent synaptic pools ands on the mechanical state of the muscle. Voluntary control of the stimulated muscle influences theseco conditions but the effects on the EMG are quite different from those on the twitch. This paper $\overrightarrow{\omega^{\prime}}$ discusses these effects under isometric conditions.

The Hoffmann reflex (H-reflex) is a monosynaptic reflex induced by electrical stimulation of Ia spindle afferent fibres at intensities below the thresholds of the motor fibres (Hoffmann, 1918; 1922; Magladery and McDougal, 1950; Magladery, Porter, Park, and Teasdall, 1951). It is most easily measured, in man, from the soleus muscle when the tibial nerve is stimulated where it crosses the popliteal fossa.

The reflex is manifest electrically and mechanically. Approximately $30 \mathrm{msec}$ after the stimulus, a synchronized burst of electromyographic (EMG) activity, the H-wave, is recorded and almost immediately thereafter, the gastrocnemius and soleus muscle tensions begin to rise. This twitch tension reaches its peak in approximately $120 \mathrm{msec}$ and then falls. Figure 1 shows such a response.

Both the H-wave and the twitch are dependent, not only on the intensity of the electrical stimulus, but on the static ${ }^{1}$ initial conditions at the ankle joint-that is, initial foot torque (IFT) and initial foot angle. These effects are sometimes used clinically to facilitate the tendon jerk which is a closely related reflex. The effects of IFT on the $\mathrm{H}$-wave have been described qualitatively by several investigators (Hoffmann, 1922; Paillard, 1955; Hagbarth, 1962) but the interactions between the four variables have not been thoroughly described. In this paper we shall discuss the effects of IFT on both the $\mathrm{H}$-wave and the twitch torque at different stimulus intensities. In a subsequent paper we shall discuss the effects of foot angle.

${ }^{1}$ During time varying conditions, there are additional influences (Granit, 1955; Mark, Coquery, and Paillard, 1968; Gottlieb, Agarwal, and Stark, 1970).

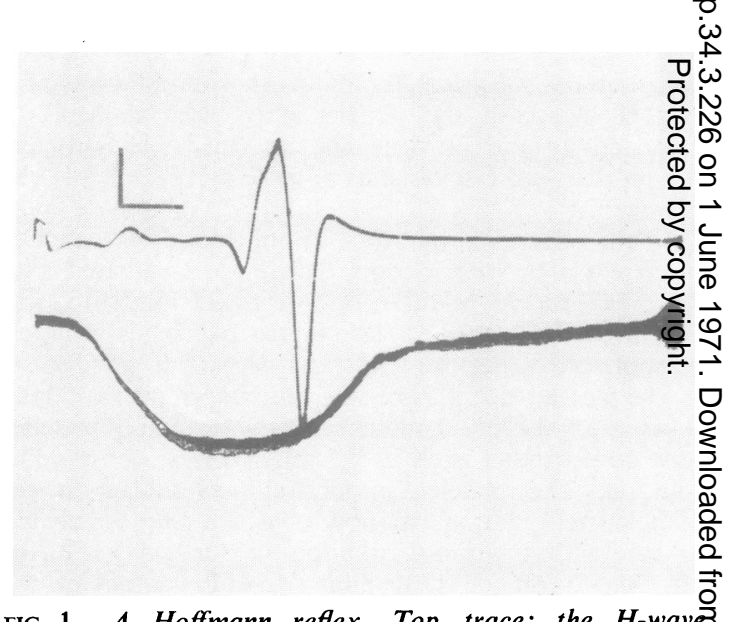

FIG. 1. A Hoffmann reflex. Top trace: the $H$-wave follows the stimulus by about $30 \mathrm{msec}$. Scales: $1 \mathrm{mV} /$ unit 10 msec/unit. Bottom trace: foot twitch torque. Scaleș. $0.15 \mathrm{~kg} \mathrm{~m} / \mathrm{unit}, 50 \mathrm{msec} / \mathrm{unit}$. (Five traces superimposed

\section{METHODS}

Our experiments were performed on nine normal male adults ranging in ages from 21 to 36 years. A subject was seated normally in a chair with his right leg extended, thes knee slightly flexed, and the foot strapped to a fixed plate: with an attached strain gauge bridge for measuring iso metric torque. The signal from this bridge was also used to provide a visual reference to help the subject maintain a constant IFT before stimulation. Differential EMCW surface electrodes $(1 \mathrm{~cm}$ diameter) were placed on the 
centreline of the lower soleus muscle about $3 \mathrm{~cm}$ apart and $20 \mathrm{~cm}$ above the base of the foot, and another pair was placed on the anterior tibial muscle. A ground electrode was placed over the flat surface of the tibia. Cutaneous stimulating electrodes were located posteriorly over the tibial nerve in the popliteal fossa and anteriorly just above the knee. These were held in place by velcro straps. All electrodes were coated with Sanborn Redux Creme. Figure 2 shows a schema of the apparatus.

The electrical stimuli of $1.5 \mathrm{msec}$ duration were applied from a Grass S-8 stimulator through a SIU5 isolation unit. This was triggered at 7 to 10 second intervals by an IBM 1800 computer which recorded the isometric foot torque at 250 samples per second and the EMG at 1,000 samples per second. The data were stored on magnetic tape for later analysis.

\section{RESULTS}

Figure 3 shows the covariation in $\mathrm{H}$-wave and foot twitch-torque $(\Delta \mathrm{FT})^{1}$ as the stimulus intensity was varied from below threshold to that for a near maximal $\mathrm{H}$-wave. For the three levels of initial foot torque, the correlation coefficients (r) and regression curves are given in the Table. Note that the dorsiflexion curve uverlays the neutral curve while the plantarflexion curve is well below both.

In Fig. 4 the $\Delta F T$ is plotted against the $\mathrm{H}$-wave with the stimulus held constant (three different stimulus intensities were used as indicated in the figure) and IFT voluntarily varied over a range of $\pm 0.8 \mathrm{~kg} . \mathrm{m}$. The simple proportionality of Fig. 3 is absent here.

In order to observe the effects of IFT on both the H-wave and $\triangle F T$, Fig. 5 shows the data from Fig. 4, but IFT is the independent variable and the $\mathrm{H}$-wave and $\triangle F T$ are plotted separately.

Among the several processes which play a role in

$\Delta \mathrm{FT}$ is defined as the difference in foot torque at the time of the occurrence of the H-wave and that at the peak of the twitch.

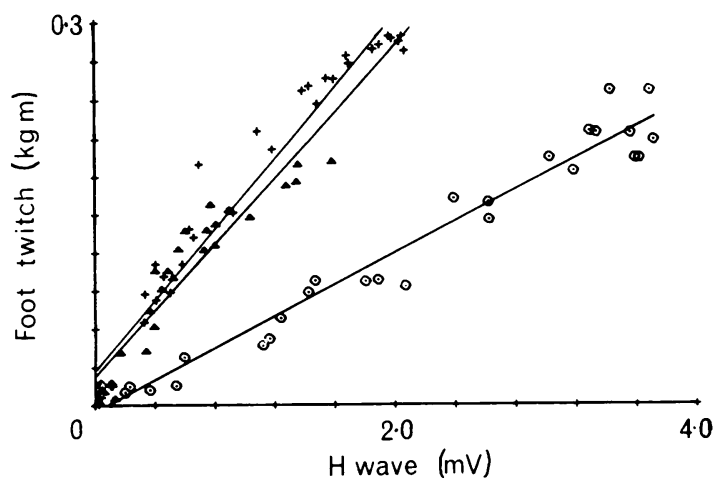

FIG. 3. Peak twitch torque as a function of peak-to-peak $H$-wave amplitude with maintained isometric foot torque. This relationship is shown at three different levels of maintained voluntary isometric foot torque as the level of electrical stimulation is varied. The three levels are indicated by: $\bigcirc$ plantarflexed, + foot at rest, and $\Delta$ dorsiflexed.

TA B LE

CORRELATION BETWEEN H-WAVE AND $\triangle \mathrm{FT}$

\begin{tabular}{lrcccr}
\hline & $\begin{array}{c}k g m \\
I F T\end{array}$ & $r$ & $\begin{array}{c}\text { Points } \\
\text { (no.) }\end{array}$ & $\begin{array}{c}k g m / m V \\
a\end{array}$ & $\begin{array}{c}k g m \\
b\end{array}$ \\
\hline Plantar & -0.61 & 0.978 & 26 & 0.064 & -0.010 \\
Neutral & 0.00 & 0.977 & 34 & 0.140 & 0.027 \\
Dorsal & 0.64 & 0.941 & 28 & 0.132 & 0.021 \\
\hline
\end{tabular}

The regression curve is $\Delta \mathrm{FT}=\mathrm{aH}+\mathrm{b}$.

this complex interaction is reciprocal inhibition of the reflex antagonist, the anterior tibial muscle, when IFT is in the dorsal direction. Reciprocal inhibition has been demonstrated in cats (Lloyd,

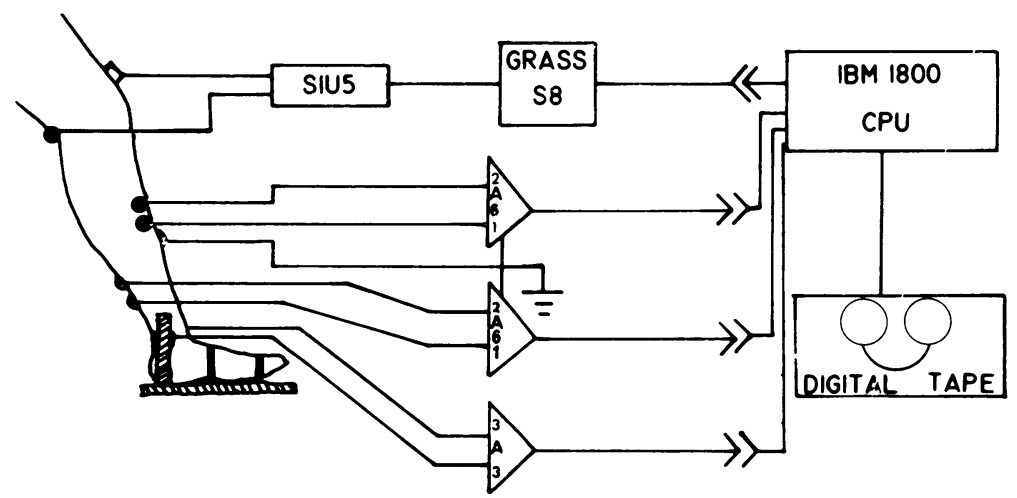

FIG. 2. Diagram of apparatus. EMGs from surface electrodes are amplified by Tektronix 2 A61 a.c. differential amplifiers with lower and upper cut off frequency settings at 60 and $600 \mathrm{~Hz}$. The strain gauge bridge output was amplified using a $3 A 3$ d.c. differential amplifier. The stimulator (Grass S8) was triggered by the digital computer (IBM 1800) at 7-10 sec intervals. 


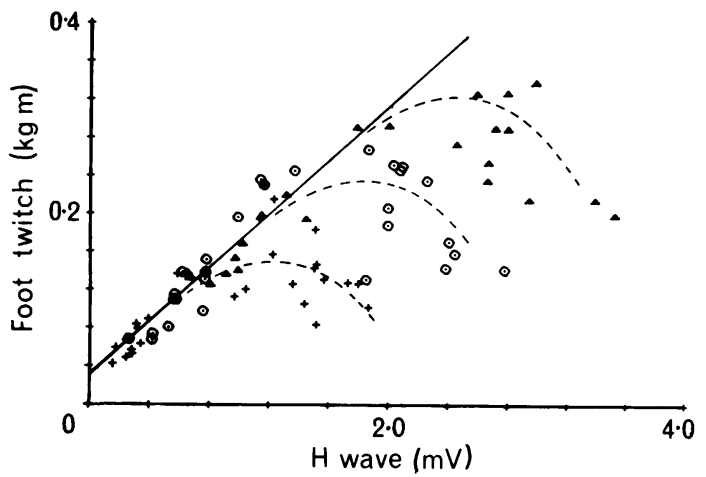

FIG. 4. Variation in twitch torque with $H$-wave when the stimulus is held constant and initial foot torque is varied. Three stimulus intensities (+ low, $\bigcirc$ higher, $\Delta$ highest). The solid line is the neutral IFT regression curve from Fig. 3 The dashed lines show the trend of deviation from that regression curve produced by plantarflexion.

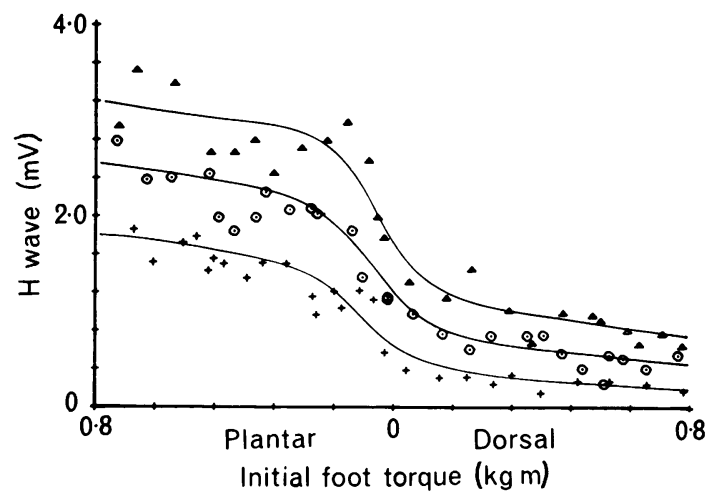

FIG. $5 \mathrm{a}$

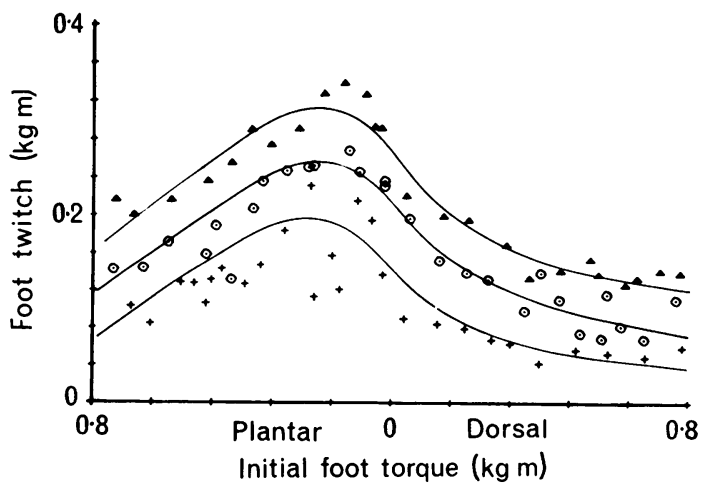

FIG. $5 b$

FIG. 5. Data from Fig. 4, redrawn with IFT as the independent variable. Variation in $H$-wave amplitude (part a) and twitch torque amplitude (part b) as IFT is varied. Three stimulus intensities. Solid lines drawn by hand show trends of data.
1943) and Hagbarth (1962) has shown its probable presence in man at high stimulus intensities-we above the threshold of the motor fibres at least- se also Liberson, 1965). Figure 6 shows the presence of this phenomenon at stimulus intensities whicf are only adequate to excite the lowest threshold fibres.

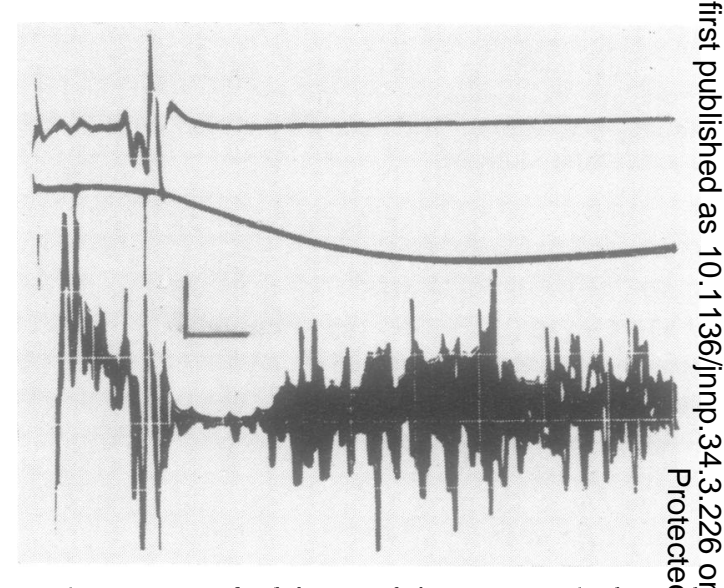

FIG. 6. Reciprocal inhibition of the anterior tibial mustle by an $H$-reflex in the soleus muscle. Top trace: $H$-wavein soleus muscle, scale $1 \mathrm{mV} /$ unit. Middle trace: foot twigh scale $0.5 \mathrm{~kg} \mathrm{~m} /$ unit. Bottom trace: anterior tibial mus EMG showing reciprocal inhibition following the sole्य $H$-wave. Dorsal IFT $0.2 \mathrm{~kg} \mathrm{~m}$. Time scale $=20 \mathrm{msec} /$ uzit (Five traces superimposed.)

\section{DISCUSSION}

The contribution of a single muscle fibre to the $\mathrm{H}$-wave is a function of the fibre's size, and its loca tion within the muscle. The former controls thet amount of current the fibre will generate, while the latter determines the effective muscle electrica列 impedance seen by the recording electrodes. The current capability should be proportional to the fibre's surface area-that is, to its radius-while the force that it generates should be proportional to its cross-sectional area-that is, to its radius squared.

Such an analysis predicts that the twitch should increase as the square of the $\mathrm{H}$-wave but this is not observed. In fact, on the contrary, in about half oㄹ. the experiments, a straight line fitted to the data for small amplitude $\mathrm{H}$-waves will be above most of the points for the large amplitude $\mathrm{H}$-waves. Clearly to explain fully the shape of the curve, the distribue tion of fibre sizes within the muscle must be knowno 
Furthermore, whether a fibre is slow or fast will greatly affect the twitch size. ${ }^{2}$

The proportionality between the $\mathrm{H}$-wave and $\Delta \mathrm{FT}$ for varying intensity stimuli shown in Fig. 3 is in full agreement with the results of similar experiments involving the tendon reflex (Clarke, 1965, 1967) and direct electrical stimulation of the motor fibre (de Jong and Freund, 1967). We are reluctant, however, to conclude from these data that there is indeed a linear relationship between the two variables. Over the limited dynamic range of inputs for which experimental data has been taken, linearity appears to be a good approximation.

The experiments cited in the references were performed with the stimulated muscle voluntarily relaxed. It is clear from Fig. 4 that the proportionality that exists, does so only under a retricted set of conditions.

The $\mathrm{H}$-wave is produced by Ia excitation of the alpha motoneurone pool and at the synapse in the anterior horn of the spinal cord it is subject to many influences (Eccles, 1967). Figure 5a shows that voluntary plantarflexion (which is the direction of the reflex twitch) could be facilitating the motoneurone pool so that more motoneurones are brought synchronously above threshold by the Ia volley. ${ }^{3}$ Conversely, voluntary dorsiflexion inhibits the motoneurone pool. In two of our subjects, strong dorsiflexion almost totally inhibited a near maximal H-wave. In the other seven subjects total inhibition with dorsiflexion could be achieved only with reduced stimuli. The amplitude of EMG activity due to voluntary effort was negligibly small and could not influence the $\mathrm{H}$-wave measurement.

Our interpretation of $\Delta \mathrm{FT}$ is necessarily more tentative than that of the EMG, since several more physiological and mechanical stages intervene between stimulus and response. Observe that from the point of maximal plantarflexion, as IFT decreases, the $\Delta F T$ increases. This increase in $\Delta F T$ is a consequence of higher incremental gain of a quiescent motor unit compared with an active one. That is, the $\Delta \mathrm{FT}$ increment caused by a single pulse in a quiescent motoneurone is likely to be greater than the $\Delta \mathrm{FT}$ increment caused by injecting a single additional pulse into a steady pulse train.

At lower levels of plantarflexional IFT, more units are quiescent and there the additional pulse caused by the stimulus results in larger twitch torque.

\footnotetext{
'There is an additional consideration in that stronger stimuli may directly excite the peroneal nerve of the anterior tibial muscle due to current spread unless great care is taken in positioning the stimulating electrode. Excitation of the anterior tibial muscle would diminish the apparent reflex twitch.

${ }^{3}$ On occasion, the $\mathrm{H}$-wave will begin to decrease at higher levels of plantar foot torque (cf. Gottlieb et al, 1970, Figure 8-1). This may represent occlusion, either at the afferent fibre at the site of stimulation or within the anterior horn of the spinal cord.
}

A second factor contributing to the $\Delta \mathrm{FT}$-H-wave relationship is the silent period in soleus muscle activity that follows the $\mathrm{H}$-wave. This period, lasting $100-200 \mathrm{msec}$ after the $\mathrm{H}$-wave, is caused by several effects: Renshaw inhibition, Golgi tendon organ inhibition, and muscle spindle disfacilitation being among them. This silent period may be viewed as a negative input (inhibition of the EMG), the amplitude being the difference between soleus activity just before the $\mathrm{H}$-wave and the activity during the silent period (zero activity) and of duration equal to the silent period. This tends to diminish the twitch torque. The effect of this transient withdrawal of voluntary input to the soleus muscle would be more pronounced at higher levels of voluntary plantarflexional effort.

In the region from small plantarflexional IFT to small dorsiflexional IFT, the $\triangle F T$ decreases because of declining facilitation and increasing inhibition of the soleus motoneurone pool, as is evidenced by the decreasing $\mathrm{H}$-wave. However, we sometimes observe that the $\Delta \mathrm{FT}$ does not continue to decrease for stronger dorsiflexion but is maintained, although the H-wave has vanished. To explain this, note that a twitch represents the summed effects of any increase in activity of the soleus muscle and decrease in activity of the anterior tibial muscle. With plantarflexion, the former element predominates, but with dorsiflexion, it is reciprocal inhibition of the anterior tibial muscle that contributes part of the twitch torque. The presence of this phenomenon is clearly shown in Fig. 6. As a result, in dorsiflexional IFT where the soleus motoneurone pool is under voluntary inhibition, the twitch partly represents the difference in anterior tibial activity before and during reciprocal inhibition. Just as inhibition of the reflex agonist muscle (silent period) diminishes the twitch torque, inhibition of the antagonist muscle increases the net measured twitch torque. Therefore, as dorsiflexional IFT increases, the effect of the diminishing $\mathrm{H}$-wave is compensated by the augmented effectiveness of reciprocal inhibition-that is, the difference in activity levels is greater.

The proportionality between the electrical and mechanical responses of a muscle to voluntary or external inputs is primarily a consequence of motor unit recruitment, which appears as a simple linearly additive phenomenon. On the other hand, when electrical stimuli are superimposed upon voluntary activity, this addition occurs, in part, within individual motor units which increase their force output, rather than solely by recruitment of additional motor units. The conversion of action potentials to mechanical force by a muscle fibre is not a linear transformation.

We may observe this in another way in Fig. 4. 
The straight line in the figure is the regression curve for the neutral IFT data of Fig. 3. Note that the data points tend to cluster about that line for neutral and dorsal IFT. This reflects the fact that dorsiflexion inhibits the $\mathrm{H}$-wave at the spinal cord but does not affect the basic EMG-twitch relationship at the muscle. Plantarflexion on the other hand, not only facilitates the $\mathrm{H}$-wave but changes that EMGtwitch relationship at the muscle. Thus, those points in plantarflexion lie below the regression curve and, in Fig. 3, the plantarflexion regression line lies well below the other two.

In general, the effects of voluntary activity are most evident at lower stimulus levels. A near maximal stimulus does not undergo much facilitation or inhibition as is to be expected with the monosynaptic reflex recruiting virtually all of the accessible motoneurone pool. By contrast, a barely suprathreshold stimulus to a relaxed muscle will appear totally inhibited by dorsiflexion and strongly facilitated by plantarflexion (cf. Gottlieb et al, 1970, Figs. $7,8,10$ ). This is in keeping with the idea that individual Ia afferent fibres have a very wide field of effect within the motoneurone pool.

This accounts for the fact that slight plantarflexion facilitates both $\mathrm{H}$-wave and twitch since the increased recruitment of motor units initially outweighs the decreasing twitch response of individual units. The weaker the stimulus, the greater is the relative facilitation of the $\mathrm{H}$-wave and the greater must be the level of plantarflexion before the two effects are equal.

It is apparent, however, that when torque or torque pulses are produced predominantly by one muscle or synergistic group of muscles, there may be a rather linear relationship between EMG and torque $\Delta F T$. On the other hand, in any mechanical response involving activation and inhibition between agonist-antagonist muscle groups, the EMG measured from any single muscle tells little, by itself, about the gross mechanical response.

\section{CONCLUSIONS}

Under conditions where the voluntary activity of the gastrocnemius-soleus muscle is held constant, a high correlation has been shown between the H-wave and twitch components of the Hoffmann reflex. Voluntary contraction of the gastrocnemiussoleus facilitates the $\mathrm{H}$-wave, while contraction of its antagonist, the anterior tibial muscle, inhibits the $\mathrm{H}$-wave. This provides a measure of the central control, exerted by the higher volitional centres, over the excitability of the motoneurone pools.

It is observed that the twitch response does not remain proportional to the $\mathrm{H}$-wave when voluntary activity changes. If a reflex is elicited in a muscle which is voluntarily contracted, the twitch if reduced, because the two activating signals summate within the same motor units rather than solely bE recruiting additional ones.

This work was partially supported by NIH Training Grant, Presbyterian St. Luke's Hospital grant-in-aid, and NSF Grant GK-17581.

\section{REFERENCES}

Clarke, A. M. (1965). Relationship between the electromyo gram and the force of the isometric reflex response of normal human subjects. Nature (Lond.), 208, 551-552.

Clarke, A. M. (1967). Effect of the Jendrassik manoeuvre oफ्छि a phasic stretch reflex in normal human subjects during. experimental control over supraspinal influences. J. Neurolo Neurosurg. Psychiat., 30, 34-42.

Eccles, J. C. (1967). The inhibitory control of spinal reflexw action. Electroenceph. clin. Neurophysiol., Suppl. No. 25 S 20-34.

Gottlieb, G. L., Agarwal, G. C., and Stark, L. (1970). Inter actions between the voluntary and postural mechanisms of the human motor system. J. Neurophysiol., 33, 365-381 Granit, R. (1955). Receptors and Sensory Perception. Gale University Press: New Haven.

Hagbarth, K.-E. (1962). Post-tetanic potentiation of myotạiß reflexes in man. J. Neurol. Neurosurg. Psychiat., 25, 1-

Hoffmann, P. (1918). Über die Beziehungen der Sehnenreffexe zur willkürlichen Bewegung und zum Tonus. Z. Biol., $\mathbf{8 8}$ 351-371.

Hoffmann, P. (1922). Untersuchungen über die Eigenreftext (Sehenenreflexe) Menschlichen Muskeln. Springer: Berłn de Jong, R. H., and Freund, F. G. (1967). Relation betwein electromyogram and isometric twitch tension in huntan muscle. Arch. phys. Med., 48, 539-542.

Liberson, W. T., Dondey, M., and Asa, M. M. (1962). Brie repeated isometric maximal exercises. An evaluation by integrative electromyography. Amer. J. phys. Med., $41 \overline{\overline{0}}$ 3-14.

Liberson, W. T. (1965). Experiment concerning reciproca inhibition of antagonists elicited by electrical stimulation of agonists in a normal individual. Amer.J. phys. Med., 44 306-308.

Lloyd, D. P. C. (1943). Conduction and synaptic transmission of reflex response to stretch in spinal cats. J. Neurophysiol. 6, 317-326.

Magladery, J. W., and McDougal, D. B. (1950) Electrophysiological studies of nerve and reflex activity in normal man. I. Identification of certain reflexes in the electromyogram and the conduction velocity of periphera? nerve fibres. Bull. Johns Hopk. Hosp., 86, 265-290.

Magladery, J. W., Porter, W. E., Park, A. M., and Teasdallį R. D. (1951). Electrophysiological studies of nerve and reflex activity in normal man. IV. The two-neurone reflex and identification of certain action potentials from spinaf roots and cord. Bull. Johns Hopk. Hosp., 88, 499-519.

Mark, R. F., Coquery, J. M., and Paillard, J. (1968). Autoo genic reflex effects of slow or steady stretch of the calf: muscles in man. Exp. Brain Res., 6, 130-145.

Paillard, J. (1955). Réflexes et Régulations d'Origine Proprioceptive chez l'Homme. Libraire Arnette: Paris.

Táboříková, H., and Sax, D. S. (1968). Motoneurone pooß̂ and the H-reflex. J. Neurol. Neurosurg. Psychiat., 31, 354 361 . 